\title{
La transformación digital en los métodos y temas de la investigación brasileña de Información y Documentación 2010-2019
}

\author{
Francisco Carlos Paletta*, José-Antonio Moreiro-González** \\ *Universidade de São Paulo, Departamento de Biblioteconomía y Documentación. Universidad Carlos III de Madrid \\ Correo-e: fcpaletta@usp.br | ORCID iD: http://orcid.org/0000-0002-4112-5198 \\ **Departamento de Biblioteconomía y Documentación. Universidad Carlos III de Madrid \\ Correo-e: jamore@bib.uc3m.es ORCID | iD: http://orcid.org/0000-0002-8827-158X
}

Recibido: 23-03-20; 2a versión: 24-04-20; Aceptado: 07-05-20; Publicado: 04-05-2021

Cómo citar este artículo/Citation: Paletta, F. C.; Moreiro-González, J. A. (2021). La transformación digital en los métodos y temas de la investigación brasileña de Información y Documentación 2010-2019. Revista Española de Documentación Científica, 44 (2), e293. https://doi.org/ 10.3989/redc.2021.2.1763

Resumen: A partir de la base de datos Social Sciences Citation Index de WoS se identifican los artículos científicos brasileños que atienden a temas y métodos relacionados con la transformación digital en Información y Documentación, en el intervalo 2010-2019. Con los títulos y palabras clave de los 1039 artículos recuperados se elaboran tablas dinámicas. Luego los títulos se categorizan por expertos, mientras que para las keywords se usa el modelo de n-grams con el filtro de Fruchterman-Reingold. Sus coocurrencias se visualizan en clústeres elaborados con el método Louvain de detección de comunidades. Los resultados se exponen en tablas y gráficos comentados sobre la internacionalización, coautoría e interdisciplinariedad de los artículos, junto a las técnicas de investigación seguidas en ellos. Se estudia la progresión temática de los que se refieren al ambiente y la transformación digital. Se evidencia una aportación numerosa y significativa de los autores brasileños al estudio de los efectos que los escenarios digitales causan en el sector de la información.

Palabras clave: análisis de materias; análisis de contenido; artículos de revista; Información y Documentación; transformación digital; SSCI-WoS; Brasil.

\section{Digital Transformation in the methods and subjects of the Brazilian Library and Informa- tion Science research (2010-2019)}

Abstract: From the Social Sciences Citation Index database, those Brazilian scientific articles that address issues, methods and consequences of digital transformation into LIS, in the 2010-2019 interval are identified. Dynamic tables are elaborated with the titles and keywords of the 1039 retrieved articles. The categorization of the titles is done by experts, while for the keywords, the n-grams model is used along with the Fruchterman-Reingold filter. Their co-occurrences are visualized in clusters elaborated with the Louvain method of community detection. The results are presented in tables and commented graphics on the internationalization, co-authorship and interdisciplinarity of the articles, together with the research techniques followed in them. In The thematic progression of those that refer to the Digital environment is studied. An abundant and significant contribution of the Brazilian authors to the study of the effects that the digital scenarios cause in the information sector is observed.

Keywords: subject analysis; content analysis; SSCI-WoS; Brazil; journal articles; Library and Information Science; digital transformation.

Copyright: ( 2021 CSIC. Este es un artículo de acceso abierto distribuido bajo los términos de la licencia de uso y distribución Creative Commons Reconocimiento 4.0 Internacional (CC BY 4.0). 


\section{INTRODUCCIÓN}

Entorno y transformación digitales son dos conceptos provenientes del mundo empresarial, donde la actividad digital les da ventajas por la inmediatez, cantidad, ubiquidad y uso multimedia de información. El impulso de las Tecnologías de la Información y Comunicación (TIC) ha transcendido desde las empresas a la salud, el trabajo, los desafíos sociales, la cultura, la educación y la vida personal. Asimismo, su continuo avance afecta al intercambio continuado de bienes del conocimiento, pues de acuerdo con Fleischmann (2007), ninguna actividad es ajena a la cantidad de información accesible, ni a los cambios en su creación, difusión y uso. Aquí está la mayor diferencia de nuestro mundo con los periodos históricos precedentes, que llegan a parecernos carentes de información. Igual que sucede en las empresas, la integración de las tecnologías en todos los aspectos del funcionamiento y gestión de los centros de información hace que su intervención alcance a los procedimientos técnicos, a la atención y comunicación con los usuarios y con otros profesionales, incluso a la oferta de servicios y a los nuevos compromisos y tareas gracias al análisis de datos (Matt y otros 2015). De modo que la transformación no se limita a la explotación e integración de las TIC, pues afecta a todos los aspectos de la vida profesional y tiene consecuencias en los métodos que se emplean para investigar y trabajar.

Las consecuencias que esta transformación tiene en Library and Information Science (LIS) se deben de estudiar desde una triple perspectiva. Primero, desde la investigación que la analiza, genera nuevo conocimiento y lo aplica a la solución de los más variados problemas; pasando por la docencia para transmitir comprensión de la situación y preparar o actualizar a los profesionales en las competencias y destrezas requeridas para desarrollar sus cometidos; hasta llegar, de manera especial, al rastreo e identificación de las circunstancias y condiciones de la actuación profesional. Consideramos que investigar los efectos de la transformación digital permite percibir, antes que nada, los avances y aplicaciones consecuentes a ella. Sin duda, los investigadores son los primeros en articular con flexibilidad los conocimientos cuando la condición transdisciplinar afecta a la propia naturaleza de la información y a su empleo y manejo, lejos de cualquier división operativa y clasificatoria del conocimiento. Otra razón para indagar se deriva de que en Brasil se impulse desde 1970 la calidad de las investigaciones y su comunicación científica con la mejora de sus postgrados (Población y De-Oliveira, 2006). Lo que, sin duda, está mediatizado por el contexto digital.

\section{REVISIÓN DE LITERATURA}

Diferentes estudios argumentan el empleo del análisis de contenido para conocer las características de la producción científica por categorías académicas y por países. Desde luego, como método que cuida la selección de los documentos a analizar, el establecimiento posterior de categorías para organizar los resultados y la necesaria garantía de confiabilidad. Así lo confirman Piñuel (2002) o White y Marsh (2006). Mientras que son antecedentes del empleo de artículos de revista para conocer los temas investigados en LIS las aportaciones de Järvelin y Vakkari (1993) o de Ferrán y otros, (2017) que revelan, desde el análisis de contenido, los cambios de interés que orientan la investigación en Biblioteconomía y Ciencia de la Información. O la de Pettigrew y McKechnie (2001) para saber cómo emplean la teoría los autores de los artículos y comprobar que la mayoría de las teorías manejadas provienen de las ciencias sociales, seguido de la propia LIS, las ciencias y las humanidades. Mientras que, con el mismo fin, pero mediante análisis de cocitas, Åström (2007) estudia los cambios habidos durante quince años en los frentes de investigación, que estaban causados por la creciente dependencia de la tecnología que llevaría a LIS hacia la integración de sus diferentes espacios de investigación. Ya en el caso brasileño, Pinheiro y otros (2005) realizan análisis de contenido de los artículos de la revista Ciência da Informação, mientras que Bufrem y otros (2007) observan la temática atendida en las revistas brasileñas. Indirectamente, desde los currículos alojados en la Plataforma Lattes, Arboit-y otros (2011) estudian los artículos, libros, capítulos y comunicaciones a congresos hechos en el exterior por investigadores brasileños, para conocer el contexto de la colaboración institucional. Más cercano a nuestros propósitos se situó el estudio de la producción científica brasileña en las revistas de Información y Documentación indizadas en WoS para identificar las más relevantes (Pinto y otros, 2016).

La comunicación académica tiene que manifestar con claridad que los cambios en las líneas y temas de investigación se pueden corresponder con los principales enfoques de la práctica profesional (Hurd, 2000). Consideramos que los artículos proporcionan conceptos fundamentados sobre los que se relacionan mejor los conocimientos y habilidades requeridos en la actuación profesional, e incluso muestran términos que identifican actividades y procesos, hasta reflejar la consolidación de tendencias.

\section{OBJETIVOS}

Se quieren seguir los efectos de la transformación digital desde los artículos de publicaciones periódicas. Para conseguirlo, se propone: 
- Identificar y seleccionar la contribución de los investigadores brasileños al estudio de los entornos digitales de la información, tanto desde el punto de vista teórico como práctico.

- Percibir los enfoques con que los estudiosos analizan e interpretan la complejidad de la realidad enlazada.

- Valorar los datos del análisis para comprobar la repercusión de la información digital, así como la interdisciplinariedad e internacionalización de sus estudios.

- Desarrollar, en fase posterior, los resultados con las consecuencias que la transformación digital tiene para el mercado de trabajo y la formación universitaria.

\section{METODOLOGÍA}

\subsection{Fuente de los datos}

Se maneja información obtenida en la colección principal de la base de datos bibliográfica Social Sciences Citation Index (SSCI) de la Web of Science (WoS), a partir de una búsqueda que combina la producción en el área de Information Science \& Library Science con la limitación geográfica de Brasil como país de los investigadores participantes en la elaboración de los artículos indizados entre los años 2010 y 2019.

Utilizar los índices de citas de SSCI-WoS para analizar la productividad brasileña en Información y Documentación proporciona seguridad en la relevancia de los documentos extraídos, pues los contenidos de las revistas indizadas fueron antes revisados por pares, por lo que resulta un buen muestrario para valorar los ajustes que la economía y los procesos de digitalización han supuesto en la conceptualización, las aplicaciones y las tareas de la información (De-Oliveira, 2018). Por otra parte, los artículos de revistas indizados en WoS aseguran una comunicación científica de prestigio e impacto (Jacsó, 2009), lo que aumenta las posibilidades de aprovechamiento en los trabajos de carácter aplicado. Debido a la propagación del análisis de revistas con manifiesta calidad, SSCI es un medio oportuno de acceso a la producción en revistas de las diferentes especialidades al permitir su selección por disciplinas y aportar información sobre la adscripción institucional de todos los autores de los artículos (De-Filippo, 2013), por lo que se establece como el procedimiento preferible para comprobar el estado de la actividad investigadora en Brasil (Marteletto, 2009) a la hora de reflejar la transcendencia epistemológica y práctica que está teniendo lo digital. Que el trabajo se limite a los artículos indizados manifiesta que los resultados obtenidos no son exhaustivos respecto al objeto estudiado. Cierto es que sería aconsejable prestar atención a libros, comunicaciones, tesis doctorales y otros trabajos de fin de ciclo. Pero hacer esto sería una tarea más larga y no aseguraría la exactitud de la consulta que nos proporcionan las fuentes empleadas en este análisis.

\subsection{Procesamiento de la información}

La descarga se efectúa el día 18/06/2019. Se obtienen 1039 artículos de los que se extraen los títulos y palabras clave asignadas por los autores. No así las KeyWords Plus ofrecidas por el sistema de indización, por tratarse de palabras de interés secundario, que aparecen con frecuencia en los títulos de las referencias de los artículos, cuando una parte de nuestro análisis atiende a los propios títulos de los artículos. Además, las KeyWords Plus suelen ser palabras simples, muchas veces de carácter genérico con bastante ambigüedad. Para limpiar los títulos se contrastan con una lista de palabras vacías y otra de genéricas. Luego, los términos se fijan como n_grams, bigrams y trigrams. Los listados obtenidos se vacían en tablas dinámicas que permiten comparar el contexto de aparición de los términos en la muestra seleccionada. Para estos procesos se usa el gestor de datos Voyant (voyant-tools.org).

Ante la diversidad de las denominaciones causada por la libre asignación tanto de los títulos como de las palabras-clave $y$, para evitar las posibles indefiniciones, los términos extraídos se validan mediante contraste de experto con las preferencias y condiciones buscadas, lo que supone realizar una extracción híbrida (Vivaldi y Rodríguez, 2007). Se hace un recuento distinto para los títulos y para las palabras clave. El cálculo de aparición de éstas tiene en cuenta su mayor número o superordenación. Para precisar su significación hay que identificar los sinónimos, evitar las variaciones gráficas y reducir las variables al término canónico. Se excluyen del recuento los términos específicos cuando coinciden en el mismo título con un término genérico. Asimismo, cuando la combinación de los términos contenidos en los títulos y en las palabras clave no ofrece información decisoria respecto a su relevancia, se consultan los resúmenes de los artículos e incluso su contenido completo. Al tiempo que se comprueba el contexto en que se sitúan los términos simples. Las palabras-clave referentes a métodos y técnicas de investigación se clasifican de manera inductiva en las tablas VI y VII hasta formar las categorías inclusivas. El contexto transdisciplinar de las aplicaciones favorece que muchos términos ofrezcan una dependencia polijerárquica, y que se hayan subordinado al genérico más habitual. Mientras que la caracterización de los títulos 
dentro del entorno digital se efectúa mediante su consulta individualizada. Se seleccionan los títulos cuya materia, método o herramientas empleados corresponden al entorno digital y se disponen en categorías prácticas para ponderar su impacto.

Con los listados de términos se elaboran grafos mediante la técnica de análisis de redes sociales (SNA) (Wasserman y Faust, 1997). Los enlaces marcan la relación existente entre dos nodos cuando se utilizan simultáneamente en el mismo artículo. El grosor de los enlaces está escalado en función del número de coocurrencias que, en la base de datos, ofrece un máximo de 8 ; Como sucede con los pares redundantes bibliometría - cientometría, interdisciplinariedad - Ciencia de la Información y epistemología - Ciencia de la Información. La red se filtra con el algoritmo de Fruchterman-Reingold (Gajdoš y otros 2016) para representar solo aque- llos nodos con un peso de arista mayor o igual a 2, por lo que se eliminan los pares que aparecen solo una vez. Para calcular el modularidad de la red se emplea el Método Louvain de detección de comunidades que facilita colorear los grupos de términos entre los que existe mayor relación (Papadopoulos y otros, 2012). Finalmente, se utiliza el análisis de datos de redes sociales egocéntricas para producir los grafos de un grupo de términos.

\section{ANÁLISIS DE LOS RESULTADOS GENERA- LES DE LA RECUPERACIÓN EFECTUADA}

Las tres revistas brasileñas indizadas en JCR son las que publican más artículos (tabla I). Se destaca Informação\&Sociedade: Estudos que duplica a la que ocupa el segundo lugar, Transinformação. Estas dos revistas, junto con Perspectivas em Ciência da Informação, aportan el $69 \%$ de la muestra, 614

Tabla I. Títulos de las revistas.

\begin{tabular}{|c|c|c|}
\hline Título de la revista & ISBN & No artículos \\
\hline Informação\&Sociedade. Estudos & 0104-0146 & 342 \\
\hline Transinformação & 0103-3786 & 169 \\
\hline Perspectivas em Ciência da Informação & $1413-9936$ & 103 \\
\hline Scientometrics & $0138-9130$ & 101 \\
\hline Knowledge organization & 0943-7444 & 49 \\
\hline Investigación bibliotecológica & $0187-358 x$ & 29 \\
\hline Information processing and management & $0306-4573$ & 23 \\
\hline $\mathrm{J}$ of the association for Information science and technology & $2330-1635$ & 20 \\
\hline Journal of knowledge management & $1367-3270$ & 17 \\
\hline International journal of information management & $0268-4012$ & 13 \\
\hline Journal of informetrics & 1751-1577 & 13 \\
\hline Knowledge management research \& practice & $1477-8238$ & 11 \\
\hline Qualitative health research & $1049-7323$ & 10 \\
\hline Telematics and Informatics & $0736-5853$ & 10 \\
\hline J American society for information science and technology & $1532-2882$ & 9 \\
\hline International journal of geographical information science & $1365-8816$ & 8 \\
\hline Information development & 0266-6669 & 7 \\
\hline Journal of global information management & $1062-7375$ & 7 \\
\hline Research evaluation & 0958-2029 & 7 \\
\hline Revista Española de Documentación Científica & 0210-0614 & 7 \\
\hline Social science information & 0539-0184 & 6 \\
\hline Government information quarterly & $0740-624 X$ & 5 \\
\hline Information research & $1368-1613$ & 5 \\
\hline Information technology for development & 0268-1102 & 5 \\
\hline \multirow[t]{2}{*}{ El profesional de la información } & $1386-6710$ & 5 \\
\hline & & TOTAL 981 \\
\hline
\end{tabular}

Fuente WoS. 
artículos. La cuarta, Scientometrics, supera también las 100 apariciones y duplica en el listado a la siguiente.

En otras 35 revistas se publican cuatro artículos o menos: dos revistas con 4 artículos; otras dos con $3 ; 10$ con 2 y 23 con uno solo. Estas revistas no aparecen en el listado por razones de espacio, si bien muestran una interdisciplinariedad muy dispersa, al corresponderse muchas de ellas a dominios de colaboración menos frecuente para investigadores de LIS. A la hora de publicar es notoria la preferencia por revistas latinas, pues si a las 3 revistas brasileñas se añaden los 29 artículos aparecidos en Investigación Bibliotecológica y los 12 entre la Revista Española de Documentación Científica y El Profesional de la Información se alcanzan 715 originales, casi el $70 \%$. Todas ellas facilitan el acceso abierto a sus contenidos, aunque la última en modo diferido.

\subsection{Internacionalización, coautoría e inter- disciplinariedad}

La internacionalización se deriva tanto de las colaboraciones en la autoría de los artículos seleccionados como del idioma en que se publicaron (tabla II).

Tabla II. Idioma de publicación de los artículos

\begin{tabular}{|l|r|r|}
\hline \multicolumn{1}{|c|}{ Idioma } & No & No de citas \\
\hline Portugués & 552 & 565 \\
\hline Inglés & 429 & 2338 \\
\hline Español & 55 & 46 \\
\hline Francés & 2 & 0 \\
\hline Húngaro & 1 & 0 \\
\hline Total & 1.039 & 2.949 \\
\hline
\end{tabular}

Fuente WoS.

Se observa que los artículos escritos en inglés obtienen mayor difusión internacional (De-Souza, 2018) y, en consecuencia, son más citados. Estos datos confirman la observación de Garfield para mejorar la comunicación científica (1983).

Tabla III. Tipo de colaboración.

\begin{tabular}{|l|c|c|c|}
\hline \multicolumn{1}{|c|}{ Colaboración } & \multicolumn{1}{c|}{ No } & \multicolumn{1}{c|}{ \% } & No de citas \\
\hline Nacional & 398 & 38,30 & 1.900 \\
\hline Internacional & 293 & 28,20 & 485 \\
\hline Sin colaboración & 348 & 33,49 & 564 \\
\hline Total & 1.039 & & 2.949 \\
\hline
\end{tabular}

Fuente WoS.
La complejidad creciente de las investigaciones se afronta cada vez más en equipo, pues el $66,5 \%$ de la muestra se ha producido en colaboración (tabla III). Cuando, además, la colaboración es nacional, el 38,30\%, recibe un acusado mayor porcentaje de citas que alcanza al 64,42\%. Su desarrollo requiere la contribución de personas con competencias interdisciplinares, en especial de las áreas que se señalan en la tabla V.

Tabla IV. Índice de coautoría.

\begin{tabular}{|c|c|c|c|}
\hline Año & Autores & Artículos & Índice de coautoría \\
\hline 2010 & 266 & 107 & 2,49 \\
\hline 2011 & 274 & 106 & 2,58 \\
\hline 2012 & 304 & 128 & 2,38 \\
\hline 2013 & 225 & 79 & 2,85 \\
\hline 2014 & 257 & 93 & 2,76 \\
\hline 2015 & 257 & 97 & 2,65 \\
\hline 2016 & 392 & 130 & 3,02 \\
\hline 2017 & 369 & 133 & 2,77 \\
\hline 2018 & 324 & 121 & 2,68 \\
\hline 2019 & 161 & 45 & 3,58 \\
\hline Total & 2.572 & 1.039 & 2,77 \\
\hline
\end{tabular}

Fuente WoS.

La participación media de 2,77 colaboradores por artículo es discreta ante las variadas tareas que requiere la elaboración de los artículos de carácter aplicado, la mayoría en el listado obtenido (tabla IV). Esa participación se sitúa por debajo de la mediana de autores por documento que en España es de 3 autores en la categoría Information Science \& Library Science para el periodo 20072016 (IUNE, 2019). Sin olvidar que en un área de colaboración tan alta como Computer Science la mediana sube a 4 autores.

La interdisciplinariedad de LIS se basa en la inevitable intervención de las tecnologías informáticas y las telecomunicaciones en el tratamiento de la información. Esta circunstancia se ha fortalecido en el ambiente digital al aumentar la participación de las TIC, lo que supone abrir las actuaciones tradicionales de la información en cualquier centro o servicio de información hacia espacios mixtos (Bauer y otros, 2016), (Dias, 2008). Lo que refleja bien la tabla $V$ al destacar las áreas de Informática y de Gestión como las de mayor participación. En especial aquélla, que supone casi el $20 \%$ de las colaboraciones efectuadas por los investigadores de Información y Documentación. Continuando así la valoración que hicieron Humphreys y otros 
Tabla V. Interdisciplinariedad de los artículos

\begin{tabular}{|l|c|c|}
\hline \multicolumn{1}{|c|}{ Categorías WoS } & Frecuencia & \% \\
\hline Library and Information Science & 1.039 & 100 \\
\hline $\begin{array}{l}\text { Computer Sci. interdisciplinary } \\
\text { Applications }\end{array}$ & 118 & 11,35 \\
\hline $\begin{array}{l}\text { Computer Sci. Information } \\
\text { Systems }\end{array}$ & 83 & 7,98 \\
\hline Management & 43 & 4,13 \\
\hline Social Sci. Interdisciplinary & 17 & 1,63 \\
\hline Communication & 10 & 0,96 \\
\hline Social Sci. Biomedical & 10 & 0,96 \\
\hline Geography & 8 & 0,76 \\
\hline Geography Physical & 8 & 0,76 \\
\hline Telecommunications & 3 & 0,28 \\
\hline Health Care Sci. Services & 2 & 0,19 \\
\hline Medical Informatics & 2 & 0,19 \\
\hline Education Educational Research & 1 & 0,09 \\
\hline History & 1 & 0,09 \\
\hline History of Social Sci. & 1 & 0,09 \\
\hline Humanities Multidisciplinary & 1 & 0,09 \\
\hline Multidisciplinary Sci. & 1 & 0,09 \\
\hline Total & 1348 & \\
\hline
\end{tabular}

Fuente: WoS.

(1998) para la National Library of Medicine, en particular entre Informática, Información - Documentación y Lingüística. Y que en este caso se acrecienta, sin duda, por la aplicación de los más variados sistemas de información y gestión de TIC en empresas e instituciones.

El 4,13\% que alcanza Administración y Gestión contrasta con su notable presencia en la descripción del contenido en los títulos y palabras-clave de los artículos, que luego se atenderá. Y nos hace pensar en una participación real de investigadores de ese dominio bastante inferior a la de aplicación de los asuntos de gestión a la información y sus servicios. Otro resultado de relación escasa es el de Comunicación, dominio del que, a priori, cabría suponer una mayor colaboración (Garvey, 1979) que el $0,96 \%$ reflejado en la muestra, pues la Teoría de la Comunicación viene participando decisivamente desde que se formó el concepto de Information Science en los años 60 (Georgia Institute of Technology, 1962). Esta baja presencia reitera lo observado por Sánchez-Vigil y otros (2015) respecto al número de artículos de Comunicación en las revistas españolas de Información y Documentación, tan solo un $8,73 \%$ del total. Aunque estas cifras podrían aumentar, pues hasta 16 artículos atienden a los medios de comunicación de las re- des sociales y a los de comunicación social si se suman aquellos con perspectiva empresarial y de investigación social.

De los documentos recuperados, 55 no ofrecen palabras clave. Aunque publicaciones como Information Research, JASIST o Restaurator no las solicitan y plataformas como De Gruiter no las ofrecen en su acceso libre limitado en ocasiones a título y abstract. La media de palabras clave por documento es de 4,44 , en gran contraste entre las 17 del artículo más generoso y los que tienen tan solo una o ninguna. De las 4.373 palabras clave asignadas por los autores 2.784 solo aparecen una vez y 265 dos veces.

\subsection{Métodos y técnicas de investigación em- pleados}

La metodología y las técnicas de investigación utilizadas se declaran en los títulos o palabras-clave de 688 artículos, el 69,91\% de los 984 que ofrecían palabras clave (tabla VI).

Por su relieve se destacan las 197 presencias relativas a los estudios métricos de la información. Un 26,45\% de los artículos emplearon sus métodos de análisis, indicadores, técnicas o herramientas dentro de cualquiera de los aspectos relacionados con la descripción de la actividad y comunicación científica (Bauer y otros, 2016). El peso de estas contribuciones se entiende bien al considerar que solo en Scientometrics se publicaron 101 de los artículos. El uso de modelos estadísticos es la base de los métodos métricos de la información, potenciados además por las tareas de computación a la hora de procesar los datos (Gorbea, 1998). Pero su empleo va más allá al atender a los datos acumulados en las organizaciones, sobre todo en empresas e industrias. Como también a los derivados del procesamiento lingüístico y textual o de la recuperación de la información, en especial los de minería de textos para buscar información en grandes colecciones de documentos no estructurados (Feldman y Sanger, 2007). De modo que los métodos, modelos y algoritmos de aplicación estadística, además de estar muy unidos a los estudios métricos, se aplican en 99 ocasiones a todos los dominios de referencia de los artículos a la hora de recopilar, comparar y evaluar. Hay que considerar que el estudio métrico de la información sigue usándose con los documentos tradicionales, pero se ha abierto a los objetos de contenido electrónicos disponibles en Internet y en la web (Leydesdorff y Wouters, 1999). Sin olvidar que el entorno digital está cambiando en profundidad las formas de comunicación científica. 
Tabla VI. Métodos y técnicas cuantitativas y tecnológicas de investigación.

\begin{tabular}{|c|c|}
\hline \multicolumn{2}{|c|}{ Estudios métricos de información 197 frecuencias } \\
\hline Disciplinas & $\begin{array}{l}\text { Bibliometría 78, cientometría 27, estudios métricos 7, altmetrics } 6 \text {, informetría 4, estudios de } \\
\text { usuarios 4, webometría 2, mapas de ciencia 2, cibermetría, métricas de relevancia }\end{array}$ \\
\hline Métodos & $\begin{array}{l}\text { Análisis de citas 34, análisis de cocitas 7, indicadores bibliométricos 2, análisis de coenlaces, } \\
\text { factor de impacto, factor de impacto de las revistas, citas entre patentes }\end{array}$ \\
\hline Leyes métricas & Zipf 4, Benford 2, Bradford 2, punto de transición de Goffman 2, índice h 2, coseno de Salton, Lotka \\
\hline Herramientas & De análisis bibliométrico: Proknow c 3; redes de citaciones: main path analysis \\
\hline \multicolumn{2}{|c|}{ Análisis estadístico 99 frecuencias } \\
\hline Multivariante & $\begin{array}{l}\text { Análisis de clúster 14, modelos de ecuaciones estructurales 7, análisis multivariante 5, análisis } \\
\text { factorial 4, índice de Nihans }\end{array}$ \\
\hline Descriptivo & $\begin{array}{l}\text { Análisis de datos 9, análisis cuantitativo 8, minería de datos 3, análisis de big data, } \\
\text { indicadores analíticos del almacén de datos }\end{array}$ \\
\hline Otros & 21 casos de aparición singular: algoritmos bivariantes, genéticos, de correlación y de proximidad \\
\hline Redes y grafos & $\begin{array}{l}\text { Redes complejas 10, análisis de redes sociales 6, fusión basada en gráficos 2, gráficos y redes } \\
2 \text {, intermediación, representación de trayectoria elíptica, análisis de datos de la trayectoria, } \\
\text { similitud de trayectoria, grafos genealógicos }\end{array}$ \\
\hline \multicolumn{2}{|c|}{ Métodos de evaluación 79 frecuencias } \\
\hline $\begin{array}{l}\text { Indicadores, } \\
\text { evaluación, medidas }\end{array}$ & $\begin{array}{l}\text { Aplicados a: rendimiento, sistemas de recuperación, competencias, servicios electrónicos, } \\
\text { impacto, productividad, análisis semiótico, modelos de servicio, modelos de negocio, } \\
\text { circulación, descripción, organización; imagen o impacto social de bibliotecas, archivos, } \\
\text { museos, servicios de información; sitios web o portales }\end{array}$ \\
\hline \multicolumn{2}{|c|}{ Métodos informáticos 56 frecuencias } \\
\hline Inteligencia artificial & $\begin{array}{l}\text { Aprendizaje automático 12, programación genética 2, reconocimiento de patrones 2, } \\
\text { análisis del árbol de fallas, flujos lingüísticos formales e informales, metodología de sistemas } \\
\text { inteligentes, razonamiento espaciotemporal, red temporal }\end{array}$ \\
\hline $\begin{array}{l}\text { Procesamiento del } \\
\text { lenguaje natural }\end{array}$ & $\begin{array}{l}\text { PLN 7, Análisis de sentimiento 7, minería de textos 6, medidas de similitud 2, derivaciones, minería } \\
\text { de contenidos, n-gram, procesamiento multi-documental, reconocimiento de patrones sintácticos }\end{array}$ \\
\hline HCI & Interacción persona-máquina 3 (disciplina) \\
\hline Software & Simulación por ordenador (modelos matemáticos de ordenador) 4, computación ubicua \\
\hline
\end{tabular}

Con otras 79 presencias aparecen diferentes métodos de análisis y evaluación que aplican indicadores, evaluaciones o mediciones a los sistemas, servicios y procesos informativos. La mayoría son inseparables de los procedimientos informáticos en los que se apoyan, como sucede en la mayor parte de los apartados que estamos mostrando. Lo que no impide reconocer 56 técnicas como características de la investigación en Informática, aunque muchas de las incluidas en Inteligencia artificial sean de base estadística y las de Procesamiento del lenguaje natural muestren interdisciplinariedad inmediata con organización y representación del conocimiento (tabla VII).

Los métodos de carácter inductivo, narrativo y sintético, empleados para identificar, evaluar críticamente e integrar los resultados, en apreciación de Cooper (2003), aparecen 54 veces. La presencia de mapeo entre ellos debe de entenderse en el sentido de complemento a las revisiones sistemáticas, no como interoperabilidad (Sayão y Marcondes, 2008). Mientras que en 49 ocasiones se utiliza la investigación social para analizar los hechos de la información, obtener datos cualitativos, conocer los gustos de los usuarios o los índices de satisfacción. Este grupo se completa con las 10 presencias de análisis y cálculos de carácter geográfico, geo-morfométrico o topográfico y con el mismo número de propuestas metodológicas aplicadas a educación y formación.

Por su parte, epistemología, paradigmas y filosofía de LIS resalta en 41 ocasiones la función de la teoría en la investigación. Son cercanas las 19 apariciones de técnicas y teorías relacionadas con el análisis y la interpretación histórica de los hechos documentales o de la evolución de los temas de investigación.

Destaca el empleo de métodos y técnicas propias de la investigación en administración y economía de la empresa, junto a la gestión de sistemas de información, en especial los contables, con presencia en 65 casos. Finalmente, en 60 ocasiones se aplican métodos y técnicas semánticas e interpretativas a la representación y organización del conocimiento. Son, sobre todo, técnicas de recu- 
Tabla VII. Métodos y técnicas cualitativas de investigación.

\begin{tabular}{|c|c|}
\hline \multicolumn{2}{|l|}{ Métodos inductivos, narrativos y s } \\
\hline \multicolumn{2}{|c|}{$\begin{array}{l}\text { Estudio de caso } 17 \text {, mapeo sistemático } 15 \text {, evaluación cualitativa } 13 \text {, modelos conceptuales } 8 \text {, mediación cultural } 6 \text {, } \\
\text { revisión sistemática de la literatura } 4 \text {, método de ordenación }\end{array}$} \\
\hline \multicolumn{2}{|l|}{ De Ciencias Sociales 49 frecuencias } \\
\hline \multicolumn{2}{|c|}{$\begin{array}{l}\text { Investigación mediante encuestas } 13 \text {, análisis de contenido } 10 \text {, grupos focales } 6 \text {, paradigmas y modelos sociales } \\
5 \text {, protocolo verbal } 4 \text {, entrevista cualitativa } 3 \text {, interaccionismo simbólico } 3 \text {, triangulación metodológica } 2 \text {, teoría del } \\
\text { actor-red, enfoque narrativo, netnografía }\end{array}$} \\
\hline \multicolumn{2}{|c|}{ Geográficos y Topográficos 11 frecuencias } \\
\hline \multicolumn{2}{|c|}{$\begin{array}{l}\text { Cálculo Geomorfométrico 3, análisis de usos del suelo 2, mapas de riesgos 2, doble ambigüedad cartográfica, } \\
\text { métodos de reconstrucción empírica, estudio cartográfico, análisis espacial }\end{array}$} \\
\hline \multicolumn{2}{|l|}{ Educación e instrucción 10 frecuencias } \\
\hline \multicolumn{2}{|c|}{$\begin{array}{l}\text { Análisis de colaboración } 2 \text {, modelos de objetos de aprendizaje 2, diagnóstico del alumno, integración entre teoría y } \\
\text { práctica, método integrador, ludificación, método de proyectos, tutoría }\end{array}$} \\
\hline \multicolumn{2}{|l|}{ Administración y gestión 65 frecuencias } \\
\hline \multicolumn{2}{|c|}{$\begin{array}{l}\text { Analítica empresarial } 13, \text { indicadores de gestión } 11 \text {, modelos y procesos de toma de decisiones } 9 \text {, sistemas difusos } \\
\text { de apoyo a la decisión 5, mapas de competencias } 3 \text {, análisis de riesgos } 3, \text { cadena de suministro } 3 \text {, modelo de } \\
\text { madurez de capacidades } 3 \text {, comportamiento planeado } 2 \text {, adhocracia, benchmarking, análisis de eficiencia, FMEA, } \\
\text { modelo de gravedad, gestión de ideas, modelo integrado de gestión del conocimiento, modelo de gestión, expansión } \\
\text { multimodal de consultas, cambio organizacional, regresiones aparentemente no relacionadas, isoperms de Sebera, } \\
\text { modelo de aceptación de tecnología }\end{array}$} \\
\hline \multicolumn{2}{|c|}{ Organización y representación del conocimiento 60 frecuencias } \\
\hline \multicolumn{2}{|c|}{$\begin{array}{l}\text { Métodos y técnicas semánticas: análisis del discurso } 12 \text {, análisis de dominios 7, selección de frases nominales } 6 \text {, } \\
\text { análisis de textos 4, análisis facetado } 2 \text {, aparato formal de la enunciación, semántica formal, compatibilidad semántica } \\
\text { Recuperación de información: expansión de la búsqueda 2, procesamiento de la búsqueda 2, métodos asociativos, } \\
\text { método de búsqueda semiótica, modelo de categorización, modelo vectorial. } \\
\text { Desarrollo de vocabularios: Desarrollo de taxonomías u ontologías 9, interoperabilidad por mapeo } 2 \text {, estándares de } \\
\text { metadatos 2, SKOS 2, matriz del lenguaje, Protégé, OWL }\end{array}$} \\
\hline \multicolumn{2}{|l|}{ Fundamentación teórica } \\
\hline Epistemología de LIS 41 frecuencias & $\begin{array}{l}\text { Epistemología } 26 \text {, filosofía de LIS } 8 \text {, paradigmas } 5 \text {, } \\
\text { postura del pensamiento jurídico, método dialéctico }\end{array}$ \\
\hline Historiografía 19 frecuencias & Métodos históricos y sociohistóricos 19 \\
\hline
\end{tabular}

peración y de desarrollo de vocabularios, estrechamente relacionadas con la Informática, en especial con la Lingüística computacional aplicada al PLN, y con la estadística.

Para las fronteras de los métodos del conocimiento seguidos en un área tan pequeña como LIS no es ningún obstáculo que esta visión presente tanta permeabilidad como ya reflejara Cronin (2012).

\section{ANÁLISIS Y DISCUSIÓN DE LOS RESULTA- DOS DESDE LA TRANSFORMACIÓN DIGITAL.}

\subsection{Subcategorías de los títulos del corpus.}

Se comprueba que 510 títulos se corresponden con la transformación digital (tabla VIII), casi el $50 \%$ de la muestra, una vez contrastados los no pertinentes. Se distribuyen en categorías inducidas durante las tareas de identificación y selección, sin poder evitar el influjo de las redes de términos que se estaban elaborando con las palabras clave.
Una primera consideración proviene del uso no siempre preciso de datos, información o conocimiento como palabras clave. Al revisarse los contenidos se verifica que en ocasiones se manejan como equivalentes (Liew, 2007). En especial con

Tabla VIII. Categorías de los títulos

\begin{tabular}{|l|r|c|}
\hline \multicolumn{1}{|c|}{ Categorías } & \multicolumn{1}{c|}{$\begin{array}{c}\text { No } \\
\text { Títulos }\end{array}$} & \% \\
\hline $\begin{array}{l}\text { Centros y servicios de información } \\
\text { (InCS) }\end{array}$ & 122 & 23,92 \\
\hline Internet-Web (InWeb) & 94 & 18,43 \\
\hline Gestión del conocimiento (GC) & 83 & 16,27 \\
\hline Sociedad digital (DG) & 78 & 15,29 \\
\hline $\begin{array}{l}\text { Tecnologías de la información y } \\
\text { comunicación (TIC) }\end{array}$ & 71 & 13,92 \\
\hline Organización del conocimiento (OC) & 62 & 12,15 \\
\hline Total & 510 & 99,98 \\
\hline
\end{tabular}


su uso indistinto como elementos componentes de organización del conocimiento y representación del conocimiento, que dividen su aparición con organización de la información y representación de la información, con ligera ventaja para las primeras de cada pareja.

En primer lugar, los títulos incluidos en la categoría Centros y servicios de información son un reflejo directo de la digitalización de los objetos de contenido y de las consecuencias que ha tenido en las formas de hacer su tratamiento, acceso y conservación. 24 títulos recorren todo tipo de archivos, bibliotecas y museos digitales, en los que la comunicación de los más variados objetos digitales depende de los ordenadores en red, en 11 ocasiones. En especial desde los problemas abordados por el movimiento abierto. Aunque ciencia abierta no sale ninguna vez en el listado de términos, si lo hacen y mucho los elementos que se integran en sus principios y recursos de conocimiento. Otros 12 títulos se ocupan de los artículos científicos digitales, las revistas electrónicas y hasta de magazines virtuales. Los medios sociales se especifican en Twitter, Orkut, Flickr, Instagram, Youtube, sobre todo en Facebook, para difundir informaciones, pero también con la presencia de blogs y hasta de likes para valorar lo emitido. Son muchos los centros que utilizan estos medios sociales para promocionar o comercializar sus actividades y recursos multimedia, en una iniciativa emprendida por los profesionales de la información para realizar su primer papel, el de comunicar. Sin olvidar los 20 títulos alrededor de los repositorios institucionales digitales que proporcionan la mejor plataforma para archivar contenidos propios, datos de proyectos u objetos de aprendizaje, con beneficio inmediato para los usuarios cuyo comportamiento en la búsqueda y uso de la información también se considera. Igual que interesan los problemas de la preservación digital o de las publicaciones fraudulentas. Es notable el avance que ha habido desde los trabajos iniciales, casi veinte años atrás, cuando se centraban en los procesos de digitalización de los acervos custodiados en soportes físicos.

Por su parte, los títulos incluidos en TIC y en Internet-Web son inmediatos a los aspectos transdisciplinares de investigación, aplicación y funcionalidad intermediados por lo digital, pues casi siempre sobrevuelan las investigaciones conjuntas de LIS con otros dominios. En especial los de Internet-Web cuyas apariciones son complicadas de deslindar, pues interfieren con cualquier ámbito de aplicación. Si agrupamos los 189 títulos válidos de ambas se percibe con claridad la importancia de los factores tecnológico-digitales en LIS. La contribución de los sistemas y procesos digitales es imprescindible para los centros de información, pero más aún para ges- tionar el conocimiento en empresas y organizaciones. La categoría de Internet-Web incluye 94 documentos que abarcan todo el espectro de internet desde red informática hasta internet de las cosas, junto a la información que permite compartir y que alberga la web. Con presencia acusada de redes sociales, de cada una de sus aplicaciones y herramientas colaborativas, y de medios electrónicos. También en este caso se percibe el empleo confuso de redes sociales en el sentido de sociedad en red o de comunidad virtual frente a apariciones que la relacionan con estudios de producción científica. En los títulos referentes a la web se da mucha atención a la web 2.0 y sus recursos; a la web semántica, sus motores, tecnologías y especificaciones; a la arquitectura de la información, protección de datos, big data, datos enlazados y datos abiertos; búsquedas y navegación en la web, web invisible y sitios web de museos.

Las categorías gestión del conocimiento y organización del conocimiento reflejan la incidencia de la intervención tecnológica y los cambios habidos en la gestión y en el tratamiento del conocimiento y de los objetos de contenido. Sin duda, las empresas e instituciones emplean la capacidad gestora que les ofrecen las TIC para potenciar el valor económico del conocimiento en su proceso productivo y organizativo (Risso, (2012). Por lo que dependen cada vez más del manejo tecnológico para favorecer su agilidad, su adaptación a los procesos en red y la gestión de su conocimiento (Del-Canto y otros, 2014). El grupo de 83 títulos de la categoría gestión del conocimiento tiene que ver, por una parte, con investigaciones focalizadas en el empleo de técnicas de la información para e-commerce, portales de negocios, portales de empresa, conocimiento tácito, toma de decisiones y márketing, como identificaran Kroll y Pinto (2018). Por otra, los modelos de gestión del conocimiento se aplican de manera creciente al buen desempeño de los sistemas y centros de información. En especial a los procedimientos destinados a satisfacer la atención que, cada vez más individualizada, exigen los usuarios. Entre otras razones, porque la implicación del márketing mejora la prestación de servicios para atender las solicitudes de los usuarios, así como para conocer la satisfacción que alcanzan (Do-Amaral, 2011). En este sentido, son apreciables los 12 títulos referentes a gestión en las bibliotecas y a calidad de los servicios de información, o los 8 de márketing de información digital. Asimismo, se destaca la presencia del conocimiento compartido y la transferencia de conocimiento. En relación con la estrategia digital y la gestión de negocio no se muestran, como sería de esperar, referencias a SEO, cuando Brasil es el país de América latina con mayor empleo de estos 
especialistas (Romero, 2019); ni a construcción de enlaces, análisis web o proyectos web. Lo que indica que su atención se deja en manos de Administración y gestión o de Comunicación. En los perfiles combinados de estos ámbitos no se identifica ninguna aparición de gestión de comunidades, gestión de contenidos digitales o edición de contenido en el sentido que les da FTI-AMETIC (2012). Sí hay bastantes de medios de comunicación de las redes sociales, hasta 12 . Muchas de ellas en aplicaciones métricas al estudio de sus contenidos, junto a su empleo en el márketing digital de la información o de las bibliotecas antes referido (Dueñas y otros, 2005). De nuevo se manifiesta la escasa investigación conjunta entre sectores que deben de colaborar ante la ausencia de estudios transdisciplinares de TIC, LIS y Comunicación (Moreiro, 2018).

No todos los contenidos y técnicas atendidas por los títulos de gestión y de organización del conocimiento pueden entenderse siempre dentro del entorno digital. Por lo que no se contabilizaron 73 de los artículos cuyo tema les es propio pero que presentan continuidad temática, técnica o metodológica con los usos precedentes, como sucede también al grupo de Organización del conocimiento (OC) y, en realidad, a todas las categorías.

El rango de la Sociedad digital hace referencia inmediata al impacto de las TIC en la cultura, el desarrollo de las ciudades, el e-gobierno, el empleo, la ciencia, la educación y la organización social. Se muestra en tres marcos de representación. El primero en torno a las consecuencias inmediatas que ha tenido la transformación digital a través de denominaciones variadas: postmodernidad o era digital, sociedad del conocimiento, sociedad red, ciberespacio o cultura digital. El segundo en torno al empleo de internet para proporcionar información y servicios públicos o para facilitar la comunicación y la colaboración entre el gobierno y los ciudadanos sobre las que apoyar la planificación y la toma de decisiones, con atención al uso de internet en la administración, las ciudades o los colectivos inteligentes mediante la democracia y la participación electrónicas, los movimientos sociales en línea o el e-gobierno. Por fin, los artículos que se han elaborado en torno a los aspectos sociológicos de las redes sociales y los comportamientos de las nuevas generaciones. Aparecen bien representados los aspectos tocantes a la inclusión digital, también a la brecha digital. De otros campos, tienen especial repercusión por su número, los títulos interdisciplinares sobre e-Salud y tecnología asistencial, junto a los de banca móvil, webs bancarias y microfinanzas, o de los móviles, hasta alcanzar a las plataformas de música, los detectores de humo de tabaco en el metro o el petróleo y el gas. Con presencia de los problemas éticos y jurídicos causados por el uso indebido de lo digital, en especial el plagio y las copias fraudulentas. Llama la atención la representación relacionada con educación desde las habilidades y derechos básicos en competencias de información y formación permanente. Y desde luego, los entornos virtuales de aprendizaje, los objetos de aprendizaje y la educación a distancia. Sobre formación y enseñanza en LIS hay otro grupo significativo, pero está poco relacionado con los ambientes digitales, fuera de su modalidad de impartición a distancia.

La clase TIC agrupa 71 títulos, entre los que aparecen en más de diez ocasiones el propio término TIC (De-Araújo y Oliveira, 2015), junto a recuperación de información y organización e industria de TI. Con más de cinco se sitúan visualización de información; inteligencia artificial; motores y robots de búsqueda; ingeniería del conocimiento; uso, transferencia y convergencia de la tecnología; y desarrollo e industria de programas. Junto a una gran variedad de menor presencia que va desde profesional de TI o nanotecnología, pasando por redes cooperativas, ética de la información digital, lectura digital o infografía, hasta TI inalámbrica o ciber-infraestructura de investigación, que permiten representar con rigor el papel de las aplicaciones tecnológicas en LIS.

A los resultados del análisis de Organización del conocimiento se les da mayor atención al atender luego a los clústeres de términos. Su contexto es inseparable de la vinculación a la Web semántica, con la vigencia de los nuevos estándares o, incluso, de los vocabularios y esquemas elaborados por empresas e industrias para organizar y gestionar su información. Adquieren ahí un vigoroso espacio las ontologías, con 24 apariciones, junto a 9 de las taxonomías. Sin olvidar los 37 artículos correspondientes a Sistemas de organización del conocimiento (SOC) y vocabularios semánticos.

\subsection{Clúster de las palabras-clave de autor}

El clúster de las palabras clave contenidas en los 984 documentos representa todo el léxico utilizado por los autores para indizar sus artículos. Por lo que incluyen muchos conceptos comentados antes al tratar la metodología utilizada en 688 de los artículos. El tamaño de los nodos depende del grado de enlaces de un término con los del resto del grafo. Mientras que el grosor de los enlaces está escalado en función del número de apariciones simultáneas de las palabras clave en el mismo artículo. El grosor de las aristas en la base de datos va de 1 a 8 , el máximo número de veces que un par de palabras clave coinciden. Como, por ejemplo, en los pares antes señalados de bibliometría - cientometría; interdisci- 
Figura 1. Red de palabras clave de autores

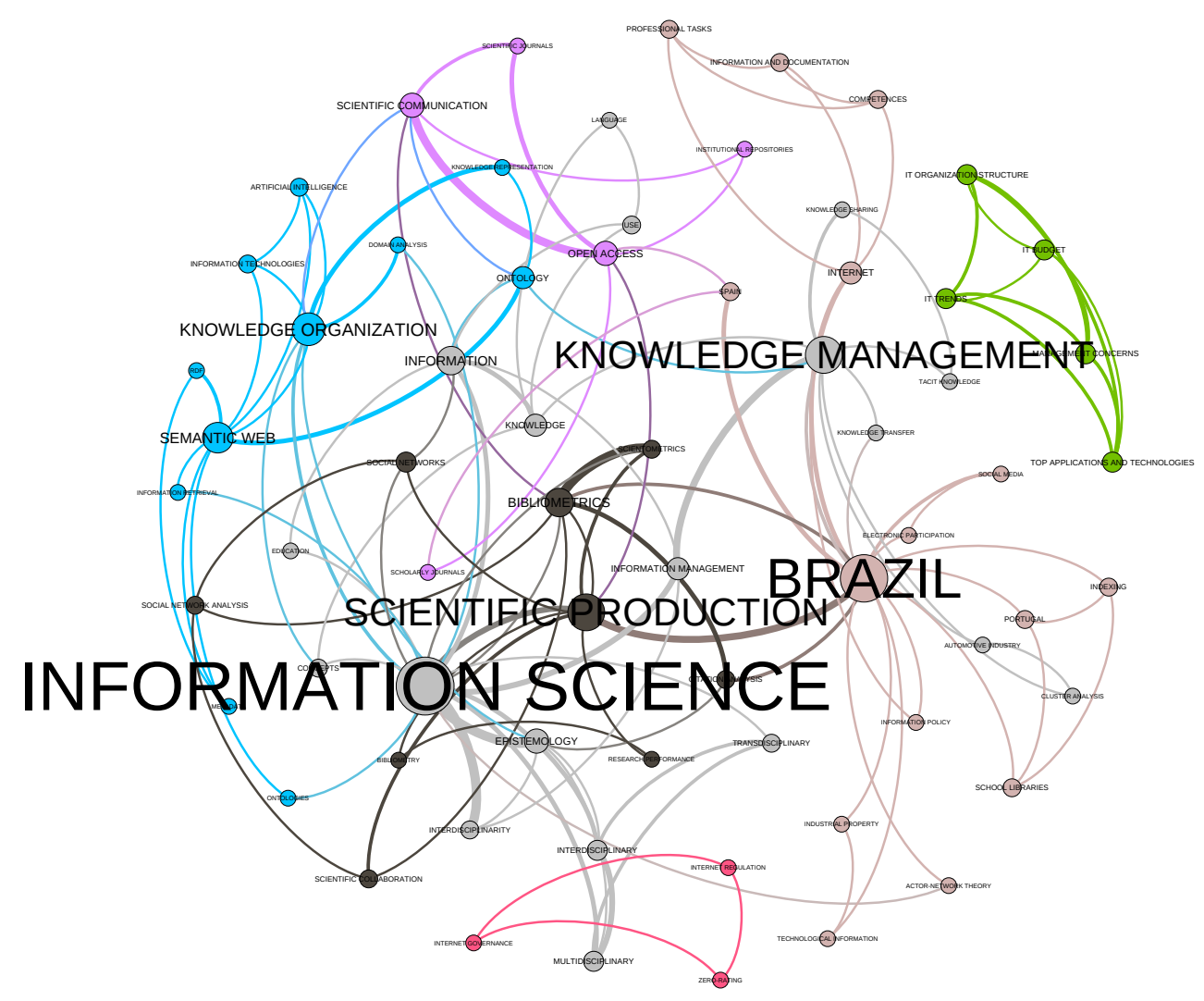

plinariedad - Ciencia de la Información y epistemología - Ciencia de la Información, todos ellos con 8. La red se compone de 2784 términos y 8031 pares de términos directamente relacionados con el ámbito digital. Lo que supone el $43,82 \%$ de las palabras clave asignadas a los artículos. En un porcentaje que resulta cercano al obtenido en el caso de los títulos. Sin embargo, la acumulación final de tan alto número de nodos y aristas presenta muchas dificultades de consulta por su casi imposible visualización. Por lo que se recortó para representar solo aquellos nodos con un peso mayor o igual a 2 .

La red de la gráfica 1 ofrece 67 nodos, el 2,41\% de los 2784 nodos totales, y 129 aristas, de las que se ven aquí solo el $1,61 \%$ de las 8031 relaciones posibles. Los términos centrales del clúster son Information Science que muestra 22 enlaces en esta gráfica y 197 en toda la red; y Brazil con 17 y 196, respectivamente (tabla IX). Ambos son dos de los términos de la búsqueda realizada en WoS. Por lo que se atiende al análisis de las redes formadas por el resto de los términos de la tabla, para reflejar los temas de investigación más afianzados. En esta red los términos y relaciones de mayor empleo son:
Tabla IX. Clúster de nodos y enlaces de las palabras clave de autores

\begin{tabular}{|r|l|c|c|}
\hline & Nodos & Enlaces & $\begin{array}{c}\text { Enlaces } \\
\text { totales }\end{array}$ \\
\hline 1 & Ciencia de la información & 22 & 197 \\
\hline 2 & Brasil & 17 & 196 \\
\hline 3 & Producción científica & 12 & 90 \\
\hline 4 & Gestión del conocimiento & 12 & 117 \\
\hline 5 & $\begin{array}{l}\text { Organización del } \\
\text { Conocimiento }\end{array}$ & 10 & 90 \\
\hline 6 & Web semántica & 12 & 72 \\
\hline 7 & Bibliometría & 8 & 114 \\
\hline 8 & Información & 8 & 111 \\
\hline 9 & Epistemología & 6 & 43 \\
\hline 10 & Acceso abierto & 6 & 57 \\
\hline 11 & Comunicación científica & 6 & 61 \\
\hline 11 & Gestión de la información & 5 & 73 \\
\hline 13 & Internet & 5 & 51 \\
\hline 14 & Conocimiento & 5 & 42 \\
\hline 15 & Ontologías & 5 & 39 \\
\hline
\end{tabular}


Se comprueba que cinco de los nodos con mayor frecuencia se vinculan directamente con las innovaciones tecnológicas y la transformación digital. Si bien, esa vinculación va más allá de la correspondencia directa entre nodos, al darse una proximidad contextual al resto de nodos e islas. Como muestra que tareas profesionales y competencias enlacen con Internet que, a su vez, lo hace con gestión del conocimiento. No se incluyen en la tabla, pero consiguen cuatro arcos: redes sociales, multidisciplinariedad, interdisciplinariedad y transdisciplinariedad. Las dos islas que se observan en el clúster están compuestas por los nodos: tendencias TIC para el futuro digital, presupuestos de TIC, estructura organizativa de TIC, problemas de gestión y principales aplicaciones y tecnologías, la primera; mientras que la segunda se integra por gobernanza de Internet, regulación de Internet y tipo cero. La relación de ambas islas es inmediata con las TIC. Igual que sucede con la cuasi-isla de relaciones en torno a interoperabilidad-metadatos.

El grupo de términos referentes a gestión del conocimiento está integrado por 117 pares de términos totales que suponen el $8,2 \%$, lo que habla de su relevancia (figura 2). La interdisciplinariedad en la práctica metodológica de Gestión del conocimiento se refleja no solo por los dominios de aplicación, sino también por su empleo simultáneo con TIC y con SOC. Se muestra con claridad desde los enlaces de ontología y de taxonomía. Pues ambas organizan la información e integran los nuevos datos, a partir de los cuales se toman decisiones y se realizan operaciones comerciales. De modo que una parte representa el conocimiento sobre los objetos de contenido y otra de las empresas que los sirve Su situación es intermediaria entre TIC, Web semántica y gestión del conocimiento. Este clúster enlaza a través de los nodos Información y gestión de la información con organización del conocimiento. Los nodos de ontologías, con 24 apariciones en la red total, y los 9 de taxonomías, aparecen conectados con gestión del conocimiento. También, pero con menor presencia, los 8 de tesauros y los 3 de folksonomías. Son 37 los nodos correspondientes a SOC, PLN, indización automática, metadatos, interoperabilidad y vocabularios, entre otros. Si bien con presencia en el clúster de 10 enlaces más que muestran continuidad en nombres, métodos y procesos que son ajenos al ambiente digital.

Figura 2. Clúster de gestión del conocimiento y organización del conocimiento

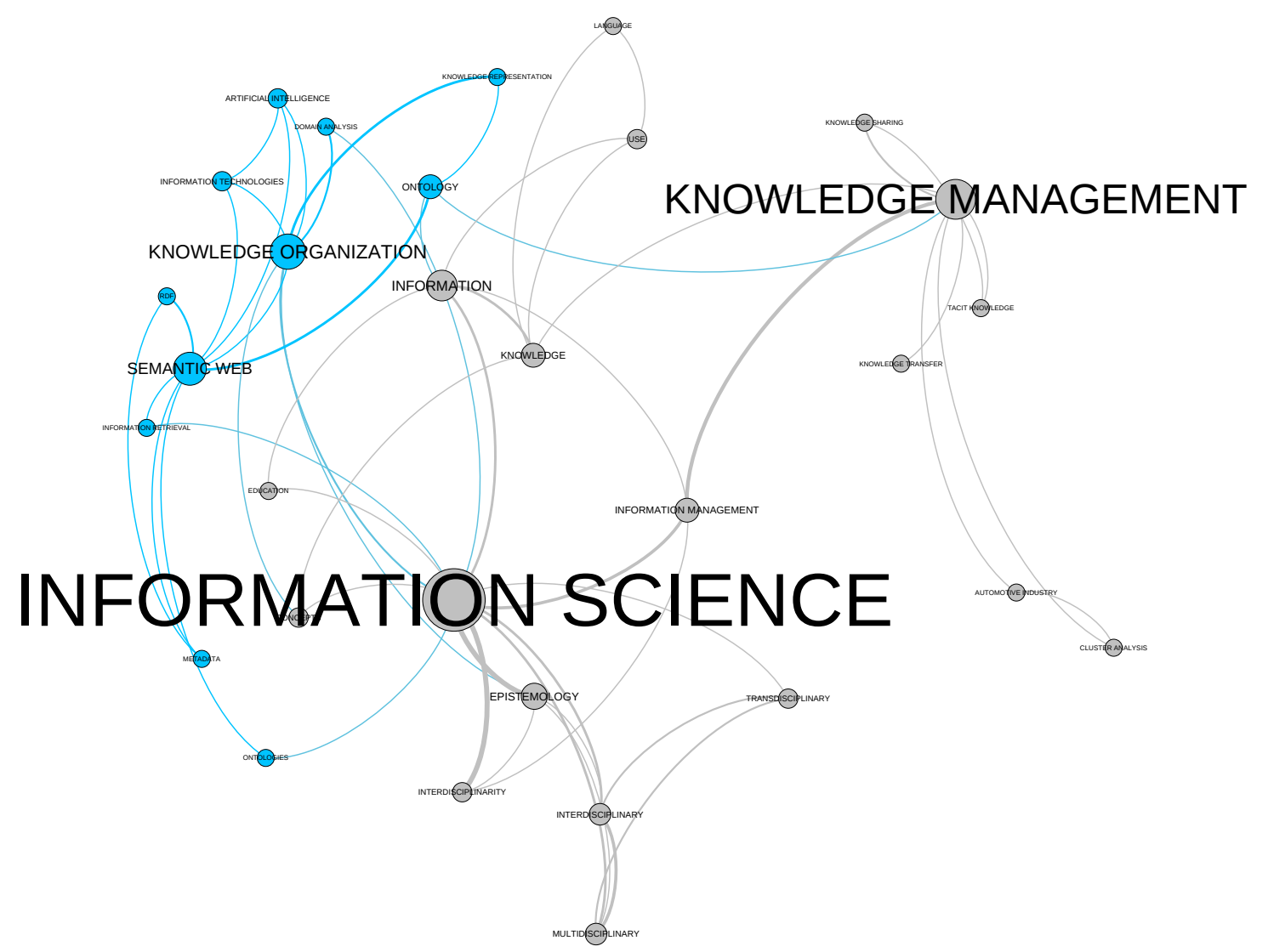


Como última consideración, destaca la presencia del nodo TIC cuya importancia se muestra en los 201 enlaces que protagoniza y en los 173 documentos que lo contienen. Si añadimos los aportados por los nodos Internet y Web que afectan a 186 enlaces, siendo el asunto principal de 71 títulos de documentos, se percibe la pujanza de los asuntos relacionados con las tecnologías que fundamentan la transformación digital.

En las palabras clave se reflejan muchos nombres de estados y ciudades brasileñas, de universidades y de otras instituciones; también de personas académicas.

\section{CONCLUSIONES}

Respecto al objetivo general, se identifica una intensa participación de los investigadores brasileños en el escenario de la transformación digital en LIS, donde han cambiado los modelos y el manejo de la información. Las herramientas y tecnologías digitales apoyan la investigación colaborativa, la extracción, análisis y asociación de los datos y su posterior visualización, comunicación y difusión. LIS, en cuanto ciencia social, además del ubicuo procesamiento informático, refleja su estrecha colaboración con los procesos estadístico-métricos, de evaluación, de administración y gestión, así como la aplicación de sus procedimientos a un sin fin de especialidades y de sectores de actuación.

Desde la finalidad del primer objetivo específico se comprueba que el carácter interdisciplinar de LIS lleva al empleo de métodos y aplicaciones variadas. Entre los que destacan los relacionados con los estudios métricos de información y los análisis estadísticos que intervienen en gran parte de las aplicaciones, más allá de las propias métricas. Igual que sucede con la intervención decisiva de los métodos informáticos. Sin olvidar las investigaciones sociales, en especial las derivadas de la Gestión-administración para diseñar, evaluar y mantener los centros y servicios. Así como las de carácter semántico y lingüístico aplicado a organización y representación del conocimiento, con el empleo tan exclusivo en LIS de las técnicas de recuperación de información, junto a otros métodos inductivos. No se confirma un número elevado de investigaciones descriptivas y exploratorias. Tal vez porque las revistas indizadas rechacen, entre otros, los manuscritos que no suponen modificación en el estado de los conocimientos.

El propósito del segundo objetivo se alcanza desde la claridad con que los artículos muestran la influencia de la transformación digital en los cambios metodológicos y en los asuntos estudiados. El enlace con la profesión es inmediato si se añade que muchos de ellos son aplicaciones prácticas. Se comprueba también desde el índice de coautoría que es comparable con el de la interdisciplinariedad necesaria para desarrollar investigaciones polifacéticas. Se muestra con claridad la colaboración con investigadores de diferentes disciplinas. Las de mayor relación son Informática de sistemas, Inteligencia artificial y Gestión-administración. Así como desde el uso de métodos de carácter automatizado, ahora potenciados por la comunicación y la capacidad tecnológica del ambiente digital, que han transformado todo el análisis y la gestión de la información y que suponen la aparición incesante de nuevos conceptos y procesos. Los sectores de colaboración identificados coinciden con aquellos en que se desarrolla la actividad profesional más reciente, pues si se categoriza se generaliza.

En consistencia con el tercer objetivo específico, es muy representativa la producción realizada en colaboración con investigadores de otros países. Los autores brasileños de LIS muestran una notable colaboración internacional, tanto por la coautoría como por el idioma de publicación. Se observa que, además del portugués, el idioma más empleado es el inglés, a mucha distancia del español. Los otros idiomas manejados, el francés y el húngaro, son testimoniales. Los artículos escritos en inglés obtienen mayor difusión internacional y, en consecuencia, son más citados.

Aunque el cuarto objetivo fija una aplicación de continuidad y, pese a que en las aplicaciones tecnológicas los procesos nunca terminan, los resultados obtenidos se deberían de considerar dentro de una línea de coherencia académica que parte de la investigación, se comunica en la docencia y se aplica en la profesión. En este caso por compromiso metodológico, inmediatez temática, preparación competencial e innovación conceptual. Quien investiga en los artículos analizados es un profesional de la información, en la mayoría de los casos profesor. De forma que habría que comprobar en trabajos posteriores si estos sectores de investigación lo son también de docencia. Si cuando se enseña se aplican las mismas competencias y habilidades que cuando se investiga. También si lo que se investiga se enseña.

Como cabría suponer, en el periodo analizado destaca la atención tocante a las colecciones de documentos digitales o digitalizados desde otros soportes, su procesamiento, preservación, conservación y curaduría, con énfasis en los servicios de acceso, gestión y procesamiento digital, en el estudio de los bancos y bases de datos, los repositorios, el acceso abierto, la organización del conocimiento y la satisfacción de las necesidades de los usuarios. La acumulación de estudios correspondientes a estos 
elementos expresa la penetración de la transformación digital en las tareas de investigación y en las actividades profesionales, si bien se observa alguna desaparición nominal como la de las bibliotecas híbridas. A la vez, se atiende al carácter relacional de la gestión y reutilización del conocimiento, sobre todo en las esferas comercial y jurídica. De hecho, el análisis de datos es uno de los resaltes, ampliado además con los big data, como consecuencia de la necesidad de emplear las TIC en las empresas, los centros de enseñanza superior y los organismos públicos para administrar, analizar y procesar datos. Siempre en relación con las técnicas estadísticas de minería, extracción de valor y análisis de corpus o con la gestión de datos de investigación. Vinculado a la experiencia en el análisis y la gestión de datos asoma, aún con cierta timidez, el uso de la inteligencia artificial, la ingeniería del conocimiento y el aprendizaje automático.

\section{REFERENCIAS}

Arboit, A. E.; Bufrem, L. S.; Moreiro-González, J. A. (2011). A produção brasileira em Ciência da Informação no exterior como reflexo de institucionalização científica. Perspectivas em Ciência da Informação, 16 (3),75-92. DOI: https://doi.org/10.1590/S141399362011000300005

Åström, F. (2007). Changes in the LIS research front: Time $\square$ sliced cocitation analyses of LIS journal articles, 1990-2004. Journal of the American Society for Information Science and Technology, 58 (7), 947-957. https://doi.org/10.1002/asi.20567

Bauer, J.; Leydesdorff, L.; Bornmann, L. (2016). Highly cited papers in Library and Information Science (LIS): Authors, institutions, and network structures. Journal of the Association for Information Science and Technology, 67 (12), 3095-3100. DOI: https://doi. org/10.1002/asi.23568

Bufrem, L.S.; Silva, H. N.; Fabian, C.; Sorribas, T. (2007). Produção científica em Ciência da Informação: análise temática. Perspectivas em Ciência da Informação, 12, (1), 38-49. https://doi.org/10.1590/S141399362007000100004

Cooper, H. (2003). Editorial. Psychological Bulletin, 129, (1), 3-9. DOI: 10.1037/0033-2909.129.1.3. https:// doi.org/10.1037/0033-2909.129.1.3

Cronin, B. (2012). The waxing and waning of a field: reflections on information studies education. Information Research: An International Electronic Journal, 17, (3), paper 529. Disponible en: http://InformationR.net/ir/17-3/paper529.html [Fecha de consulta: $18 / 08 / 2019]$.

De-Araújo, R. Ferreira; Oliveira, M. (2015). A tecnologia na pesquisa no ensino da Biblioteconomia e Ciência da Informação no Brasil: representações, conteúdos e práticas. Pesquisa brasileira em Ciência da Informação e Biblioteconomia, 10, (2), 64-72. Disponible en: http://www.periodicos.ufpb.br/ojs/index. php/pbcib/article/view/24932 [Fecha de consulta: 17/06/2019].
La misma razón subyace en la atención a la mudanza de la comunicación en empresas, administraciones y servicios de información a la hora de aprovechar la utilidad de las redes sociales para facilitar sus contenidos, servicios y tareas en beneficio de los usuarios. Otro sector de interés dinámico es el que marcan los estudios de la información en el gobierno y la democracia electrónicos, así como en las ciudades inteligentes o respecto a la enseñanza e inclusión digitales.

\section{AGRADECIMENTOS}

Trabajo financiado por el Proyecto de investigación no 2018-02595-8 de la FAPESP

\section{ACKNOWLEDGEMENTS}

This work has been funded by FAPESP Research Project No. 2018-02595-8

De-Filippo, D. (2013). Spanish scientific output in Communication sciences in WOS. The Scientific Journals in SSCI (2007-12). Comunicar, 21, (41), 25-34. [Fecha de consulta: 20/02/2020]. https://doi.org/10.3916/ C41-2013-02

Del-Canto, E.; Sánchez, P.; Martins, V. F.; Soares, A. B. (2014). Agentes generadores de innovación en las empresas: gestión del conocimiento y TIC. Revista de Auditoria, Governança e Contabilidade, 2, (3), 72-88. Disponible en: http://fucamp.edu.br/editora/index. php/ragc/article/viewFile/465/334 [Fecha de consulta: 21/04/2020].

De-Oliveira, E.T. (2018). Estudos Métricos da Informação no Brasil: Indicadores de produção, colaboração, impacto e visibilidade. Marília: Editora da UNESP.

De-Souza, C.-D. (2018). Impacto de las políticas brasileñas de ciencia y tecnología en la actividad investigadora de las universidades federales: un estudio cienciométrico del período 2003-2015. Madrid: Universidad Carlos III de Madrid [Tesis doctoral]. Disponible en: https://e-archivo.uc3m.es/bitstream/ handle/10016/27815/souza tesis 2018.pdf?sequen$\mathrm{ce}=1$ \&isAllowed=y [Fecha de consulta:11/06/2019 ].

Dias, F. S. (2008). Análise das relações interdisciplinares das pesquisas científicas em sistemas de informação. Perspectivas em Ciência da Informação, 13, (1), 199-215. Disponible en: http://www.scielo.br/pdf/pci/ v13n1/v13n1a13 [Fecha de consulta:08/08/2019]. https://doi.org/10.1590/S1413-99362008000100013

Do-Amaral, S. A. (2011). Marketing da informação: abordagem inovadora para entender o mercado e o negócio da informação. Ciência da Informação, 40, (1), 8598. Disponible en: http://revista.ibict.br/ciinf/article/ view/1327 [Fecha de consulta:11/07/2019]. https:// doi.org/10.1590/S0100-19652011000100007

Dueñas, J. C.; Burillo, V.; Ruiz, J. L. (2005). Professional ICT Profiles to Implement Services and Digital Content. Madrid: Colegio Oficial de Ingenieros de Telecomunicación. PAFET 4. Disponible en: https://www.coit. 
es/informes/pafet-4-perfiles-profesionales-tic-para-la-implantacion-de-servicios-y-contenidos-digital-0 [Fecha de consulta: 03/06/2019].

Feldman, R.; Sanger, J. (2007). The Text Mining Handbook: Advanced Approaches in Analyzing Unstructured Data. New York, Cambridge University Press. https:// doi.org/10.1017/CBO9780511546914

Ferrán-Ferrer, N.; Guallar, J.; Abadal, E. Server, A. (2017). Research methods and techniques in Spanish Library and Information science journals (2012-2014). Information research, 22, (1), paper 741. http://InformationR.net/ir/22-1/paper741.html

Fleischmann, K. (2007). Digital libraries with embedded values: Combining insights from LIS and science and technology studies. The Library Quarterly, 77, (4), 409-427. https://doi.org/10.1086/520997

FTI-AMETIC (2012). Estudio de Perfiles Profesionales más demandados en el ámbito de los Contenidos Digitales en España 2012-2017. Profesionales TIC. Madrid: Fundación de Tecnologías de la Información-AMETIC. Disponible en: https://www.redalyc.org/articulo. oa?id=93528051014 [Fecha de consulta:02/02/2020].

Gajdoš, P.; Ježowicz, T.; Uher, V.; Dohnálek, P. (2016). A parallel Fruchterman-Reingold algorithm optimized for fast visualization of large graphs and swarms of data. Swarm and Evolutionary Computation, 26, (February), 56-63. https://doi.org/10.1016/j.swevo.2015.07.006

Garfield, E. (1983). Mapping science in the third world. Science and Public Police, 10, (3), 112-127. https:// doi.org/10.1093/spp/10.3.112

Garvey, W. (1979). Communication: the essence of science: facilitating information exchange among librarians, scientists, engineers and students. Oxford: Pergamon I. Library.

Georgia Institute of Technology. (1962). Proceedings of the conference on Training Science Information specialists, October 12-13, 1961. Atlanta. Sponsored by the National Science Foundatio Dorothy M. Crosland, General Chairma 139 pp.

Gorbea Portal, S. (1998). Modelación matemática de la actividad bibliotecaria: una revisión. Investigación Bibliotecológica, 12, (24), 5-23. DOI: http://dx.doi. org/10.22201/iibi.0187358xp.1998.24.3869

Humphreys, B.; Lindberg, D.; Schoolman, H.; Barnett, O. (1998). The unified medical language system: an informatics research collaboration. Journal of the American Medical Informatics Association, 5, (1), 1-11. https://doi.org/10.1136/jamia.1998.0050001

Hurd, J. M. (2000). The transformation of scientific communication: A model for 2020. Journal of the American Society for Information Science, 51, (14), 1279-1283. https:// doi.org/10.1002/1097-4571(2000)9999:9999<::AIDASI1044>3.0.CO;2-1

IUNE. 2019. Mediana de autores por documento por categoría WoS y año (SUE 2008-2017). Madrid; IUNE. Disponible en: http://www.iune.es/es_ES/informes [Fecha de consulta:05/07/2019].

Järvelin, K.; Vakkari, P. (1993). The evolution of library and information science 1965-1985: A content analysis of journal articles. Information Processing \& Management, 29, (1), 129-144. DOI: https://doi. org/10.1016/0306-4573(93)90028-C
Jacsó, P. (2009). Five-year impact factor data in the Journal Citation Reports. Online Information Review, 33, (3), 603-14. DOI: https://doi. org/10.1108/14684520910969989

Kroll-do-Prado, J. M.; Pinto, A. L. (2018). Marketing in Brazilian Information Science: four decades of production. Informação\&Sociedade: estudos, 28, (3), 53-68. DOI: https:// doi.org/10.22478/ufpb.1809-4783.2018v28n3.37935

Leydesdorff, L.; Wouters, P. (1999). Between texts and contexts: advances in theories of citation? Scientometrics, 44, (2), 173-192. Disponible en: https:// link.springer.com/content/pdf/10.1007/BF02457378. pdf [Fecha de consulta:08/08/2019]. https://doi. org/10.1007/BF02457378

Liew, A. (2007). Understanding data, information, knowledge and their inter-relationships. Journal of knowledge Management Practice, 8, (2), 1-16. Disponible en: https://socygu.ga/1549290980.pdf [Fecha de consulta:15/08/2019].

Marteleto, R. (2009). A pesquisa em Ciência da Informação no Brasil: marcos institucionais, cenários e perspectivas. Perspectivas em Ciência da Informação, 14, (3), 19-40. https://doi.org/10.1590/S141399362009000400003

Matt, C.; Hess, T; Benlian, A. (2015). Digital Transformation Strategies. Business \& Information Systems Engineering, 57, 339-343. https://doi.org/10.1007/ s12599-015-0401-5

Moreiro-González; J. A. (2018). Reciprocidad laboral, formativa y difusora entre Información-Documentación y Comunicación social. Anuario ThinkEPI, 12, 90-95. DOI: https://doi.org/10.3145/thinkepi.2018.08

Papadopoulos, S.; Kompatsiaris, Y.; Vakali, A.; Spyridonos, P. (2012). Community detection in social media. Data Mining and Knowledge Discovery, 24, (3), 515-554. https://doi.org/10.1007/s10618-011-0224-z

Pettigrew, K.; McKechnie, L. (2001). The use of theory in information science research. Journal of the American Society for Information Science and Technology, 52, (1), 62-73. DOI: https://doi.org/10.1002/15322890(2000)52: 1<62::AID-ASI1061>3.0.CO;2-]

Piñuel Raigada, J. L. (2002). Epistemología, metodología y técnicas del análisis de contenido. Sociolinguistic Studies 3 (1), 1-42. https://doi.org/10.1558/sols.v3i1.1

Pinheiro, L. V. R.; Brascher, M.; Burnier, S. (2005). Ciência da Informação: 32 anos (1972-2004) no caminho da história e horizontes de um periódico científico brasileiro. Ciência da Informação, 34, (3), 38-49. Disponible en: http://revista.ibict.br/ciinf/article/ viewFile/1084/1187 [Fecha de consulta:27/06/2019]. https://doi.org/10.1590/S0100-19652005000300003

Pinto, A. L.; Matias, M.; Moreiro-González, J. A. (2016). Produção brasileira da Ciência da Informação na Web of Science entre 1994 e 2013 e a lista Qualis/Capes da Área. Ibersid, 10, (1), 51-61. Disponible en: https://www.ibersid.eu/ojs/index.php/ibersid/article/ view/4252 [Fecha de consulta: 08/08/2019].

Población, D. A.; De-Oliveira, M. (2006). Input e output: insumos para o desenvolvimento da pesquisa. En: Población, D. A.; Witter, G.; Da Silva, J. F. M. Comunicação \& produção científica: contexto, indicadores e avaliação. São Paulo: Angellara, 57-80. 
Risso, V. G. (2012) Aproximación teórica a la relación entre los términos gestión documental, gestión de información y gestión del conocimiento. Revista Española de Documentación Científica, 35, (4), 531554. https://doi.org/10.3989/redc.2012.4.869

Romero, E. (2019). Países de América con mayor cantidad de profesionales SEO. Karai digital. Disponible en: https://www.karaidigital.com/blog/ profesionales-especialistas-seo-america [Fecha de consulta: 18/08/2019].

Sánchez-Vigil, J. M.; Marcos-Recio, J. C.; Olivera-Zaldu; M. (2015). Producción científica sobre Comunicación y Medios en las revistas de Documentación (20002014). Revista Española de Documantación Científica. 38, (4), e108.

Sayão, L. F.; Marcondes, C. H. (2008). O desafio da interoperabilidade e as novas perspectivas para as bibliotecas digitais. Transinformação, 20, (2), 133-148. Disponible en: https://www.redalyc.org/pdf/3843/384334798002. pdf [Fecha de consulta:21/08/2019]. https://doi. org/10.1590/S0103-37862008000200002

Vivaldi, J.; Rodríguez, H. 2007. Evaluation of terms and term extraction systems: A practical approach. Terminology, 13 (2). 225-248. DOI: https://doi.org/10.1075/ term.13.2.06viv

Wasserman, S.; Faust, K. (1994). Social Network Analysis: methods and applications. New York: Cambridge University Press. https://doi.org/10.1017/CBO9780511815478

Wellisch, H. (1972). From Information Science to Informatics: a terminological investigation. Journal of Librarianship, 4, (3), 157-187. https://doi. org/10.1177/096100067200400302

White, M.; Marsh, E. (2006). Content analysis: a flexible methodology. Library trends, 55, (1), 22-45. DOI: https://doi.org/10.1353/lib.2006.0053 